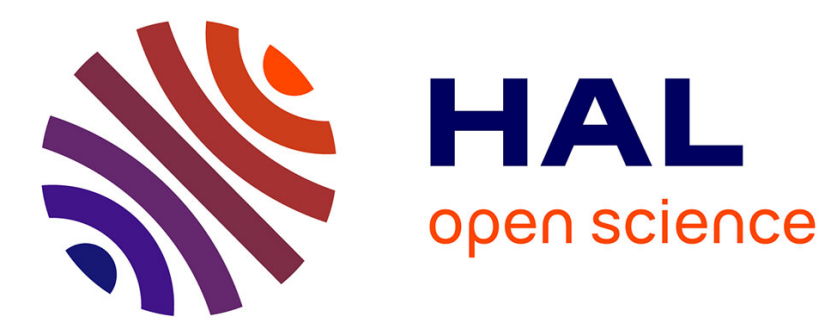

\title{
Multidimensional Characterization of Fibrillatory Wave Amplitude on Surface ECG to Describe Catheter Ablation Impact on Persistent Atrial Fibrillation
}

Marianna Meo, Vicente Zarzoso, Olivier Meste, Decebal Gabriel Latcu, Nadir Saoudi

\section{To cite this version:}

Marianna Meo, Vicente Zarzoso, Olivier Meste, Decebal Gabriel Latcu, Nadir Saoudi. Multidimensional Characterization of Fibrillatory Wave Amplitude on Surface ECG to Describe Catheter Ablation Impact on Persistent Atrial Fibrillation. 2012. hal-00718295

\section{HAL Id: hal-00718295 \\ https://hal.science/hal-00718295}

Preprint submitted on 16 Jul 2012

HAL is a multi-disciplinary open access archive for the deposit and dissemination of scientific research documents, whether they are published or not. The documents may come from teaching and research institutions in France or abroad, or from public or private research centers.
L'archive ouverte pluridisciplinaire HAL, est destinée au dépôt et à la diffusion de documents scientifiques de niveau recherche, publiés ou non, émanant des établissements d'enseignement et de recherche français ou étrangers, des laboratoires publics ou privés. 


\title{
Multidimensional Characterization of Fibrillatory Wave Amplitude on Surface ECG to Describe Catheter Ablation Impact on Persistent Atrial Fibrillation
}

\author{
Marianna Meo*, Student Member, IEEE, Vicente Zarzoso, Senior Member, IEEE, \\ Olivier Meste, Member, IEEE, Decebal G. Latcu, and Nadir Saoudi
}

\begin{abstract}
Radiofrequency catheter ablation (CA) is increasingly employed to treat persistent atrial fibrillation (AF). Nevertheless, its success is not always guaranteed, as selection of patients who could positively respond to this therapy does not rely on systematic criteria and still remains an open issue. Moreover, very little is known about the quantitative effects of this treatment over AF electrophysiology, so their quantitative evaluation is not a trivial task. In this contribution, ablation impact is quantified by a descriptor of fibrillatory wave ( $f$ wave) amplitude, so far regarded as a predictor of shortterm CA outcome. By means of principal component analysis (PCA), surface electrocardiogram (ECG) spatial diversity is exploited and contributions from all leads are combined to describe average $f$-wave peak-to-peak amplitude, whose value is automatically computed by an algorithm based on cubic spline interpolation. Our work demonstrates how CA influences fwave amplitude during the procedure as quantified by ECG inter-lead spatial variability. In addition, we show how such variations depend on procedural outcome and the duration of the postoperative blanking period.
\end{abstract}

\section{INTRODUCTION}

Persistent atrial fibrillation (AF) is an arrhythmia characterized by the generation of irregular electrical patterns, inducing an uncoordinated mechanical contraction. Several theories about its electrophysiological mechanisms have been put forward. Early experimental studies state that persistent $\mathrm{AF}$ triggering and maintenance can be explained by multiple reentrant wavelets. More recently, reentrant pathways in pulmonary veins (PVs) have been identified as AF drivers [1], whereas fibrillatory conduction passively propagates in other atrial parts. Even if radiofrequency catheter ablation (CA) has become the therapy of choice for the treatment of persistent $\mathrm{AF}$, its effectiveness is still an open issue. Its main goal is to terminate $\mathrm{AF}$ either by eliminating the trigger initiating $\mathrm{AF}$ or by altering the arrhythmogentic substrate. Since the precise pathophysiology of AF development and progression has not been completely elucidated yet, understanding whether ablation effectively suppresses abnormal rhythm sources and how

This work is partly supported by the French National Research Agency under contract ANR-2010-JCJC-0303-01 "PERSIST". Marianna Meo is funded by a doctoral grant from the French Ministry of Higher Education and Research.

* Marianna Meo, Vicente Zarzoso and Olivier Meste are with the Laboratoire d'Informatique, Signaux et Systèmes de Sophia Antipolis (I3S), Université Nice Sophia Antipolis, CNRS, France (e-mail:\{meo, zarzoso, meste\}@i3s.unice.fr).

Decebal G. Latcu and Nadir Saoudi are with the Service de cardiologie, Centre Hospitalier Princesse Grace, Monaco (e-mail: \{dglatcu, nsaoudi\}@chpg.mc). it modifies heart electrical pathways remains a challenging question. Different CA techniques have been developed, yet none of them is widely regarded as effective by the medical community. Their performance is still far from satisfactory, and they are less effective than equivalent procedures for paroxysmal AF. Since this cardiac interventional procedure is profoundly influenced by operator's subjectivity and patient's clinical background, ablation results reported by clinical centers are extremely variable and not easily comparable.

Surface standard ECG is considered a valuable tool for examining $\mathrm{AF}$, whose presence is reflected by the manifestation of irregular fibrillatory waves (f-waves) replacing the physiological $\mathrm{P}$ wave. In [2], it is shown that prolongation of atrial fibrillation cycle length (AFCL) in the coronary sinus and the atrial appendages predicts $\mathrm{AF}$ termination by $\mathrm{CA}$. In [3], reduction in AF spatio-temporal complexity following $\mathrm{CA}$ is quantified by variations in the normalized mean square error (NMSE) values between the AA signal and its rank-3 approximations on $\mathrm{V}_{1}$. The degree of $\mathrm{AF}$ organization is supposed to be directly correlated to the number and interactions of atrial wavefronts through heart substrate. Evidence reported in [4] establishes the predictive power of f-wave peak-to-peak amplitude: the higher its value, the more likely AF termination by CA. Nevertheless, ECG analysis is not always straightforward, and visual inspection is sometimes not sufficiently effective, as it cannot capture AF features underlying the whole ensemble of leads; hence, the limiting perspective of conventional methods, focusing on single leads separately.

To overcome the limitations of single-lead approaches, in [5], a novel predictor of short-term CA outcome has been developed. It describes f-wave spatial distribution and it is able to distinguish between successful and failing procedures before their execution. Such an action has been accomplished by exploiting the spatial variability typical of the standard ECG through the principal component analysis (PCA) of the AA signal. f-wave peak-to-peak amplitude is automatically computed by means of cubic spline interpolation of the AA local extrema, and the same results as those manually obtained in [4] have been reproduced. It has been demonstrated that taking advantage of the spatial diversity present in the surface ECG does not only improve the robustness to electrode selection, but it also enhances the predictive power of the amplitude parameter.

As opposed to [4], [5], we focus on variations in f-wave 
amplitude observed between the beginning and the end of the ablation. This paper presents the further ability of this multilead f-wave amplitude index to describe the myocardial electrical remodeling performed by $\mathrm{CA}$ and changes in AF pathophysiology during the procedure. This parameter highlights variations in f-wave amplitude for each patients' category, and allows the quantification of the ablation effects at different follow-up lengths. More specifically, we will demonstrate that a decline in $\mathrm{f}$-wave amplitude between the beginning and the end of the procedure can be remarked in patients experiencing AF termination by $\mathrm{CA}$.

\section{Methods}

\section{A. Experimental Protocol}

A group of 31 patients addressed for $\mathrm{CA}$ of persistent $\mathrm{AF}$ were included in this study. They all gave their informed consent. Three patients underwent a double procedure. Oneminute surface 12-lead ECG signals were acquired at the beginning and at the end of the procedure, through a standard 12-lead system at a $1-\mathrm{kHz}$ sampling rate with the aid of Prucka Cardiolab ${ }^{\mathrm{TM}}$ and Biosense $\mathrm{CARTO}^{\mathrm{TM}}$ electrophysiological measurement systems at the Cardiology Department of Princess Grace Hospital in Monaco. Ablation procedure was performed in a stepwise manner, beginning with LASSO-guided circumferential PV isolation, followed by fragmented potentials, non-PV triggers, roof line and mitral isthmus line right atrial ablation.

According to HRS Expert Consensus Statement guidelines for CA trials [6], immediately after CA performance, there is a three-month "blanking period" during which time AF episodes can occur as part of the body's healing response to the procedure. Any fibrillatory activity during this period is not taken into account. Chosen a certain follow-up time after the blanking period, success can be defined as "freedom from AF, atrial flutter or tachycardia" and discontinuation of antiarrhythmic medication. Patients participating in clinical studies should be followed at least 12 months and, at minimum, should have a 24-hour Holter monitor at three months, six months, one year, and two years.

Following these guidelines, two different criteria of procedural success have been introduced into our study. Due to the risk of recurrence of the persistent form of this disease, we distinguished a short-term success definition from a longterm one. Short-term CA outcome definition provides a preliminary perspective of the immediate effectiveness of the procedure right after a 3-month blanking period. Procedural success is regarded as $\mathrm{AF}$ conversion either directly to sinus rhythm or intermediate tachyarrhythmia, exclusively by ablation or by CA shortly followed by electrical cardioversion. According to this short-term criterion, 26 patients out of 31 experienced procedural AF termination. Longterm CA success is defined as freedom from ECG/Holterdocumented sustained AF recurrence ( $>30 \mathrm{~s}$ ) during follow up after at least six months. As far as repeated procedures are concerned, only signals acquired during the first surgical performance are analyzed. Under this hypothesis, definitive sinus rhythm (SR) restoration has been observed in 13

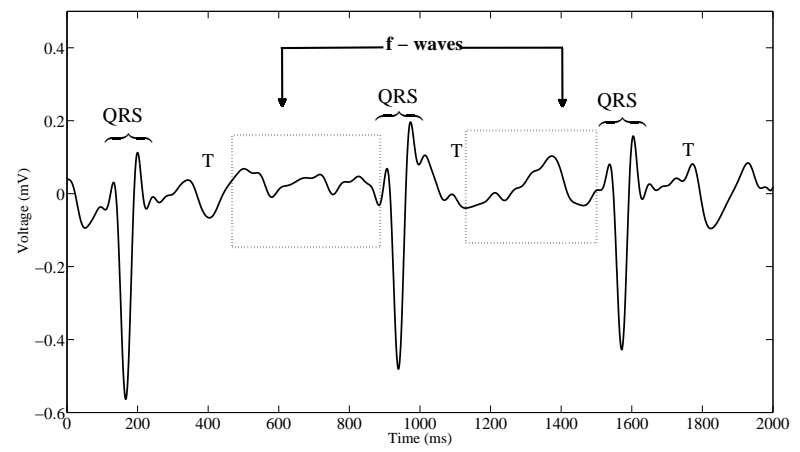

Fig. 1: Example of ECG recording during $\mathrm{AF}$ and its characteristic waves on the lead $\mathrm{V}_{1}$. Dotted boxes highlight TQ intervals which are concatenated to form the AA signal $\mathbf{Y}_{\mathrm{AA}}$ in (1).

patients out of 20. The smaller size of the second dataset is due to the fact that some ablations have been accomplished very recently, so long-term outcome is still unknown.

Owing to the risk of arrhythmic episodes and the application of complementary therapies after CA over the long-term follow up (namely, chemical or electrical cardioversion), these two criteria can return different results for the same patient.

\section{B. ECG Preprocessing and Atrial Activity Extraction}

For every patient, input ECG recordings are first processed by a fourth-order zero-phase Chebyshev bandpass filter with $-3 \mathrm{~dB}$ attenuation at $0.5 \mathrm{~Hz}$ and $30 \mathrm{~Hz}$ cut-off frequencies. This action allows the suppression of baseline wander and high frequency noise (e.g., power line interference, myoelectrical artifacts). Furthermore, this frequency interval retains the most significant $\mathrm{AF}$ content, whose dominant frequency ranges between 3 and $12 \mathrm{~Hz}$. Then, R-wave time instants are detected on $\mathrm{V}_{1}$ by Pan-Tompkins' algorithm [7]. $\mathrm{Q}$ wave onset and $\mathrm{T}$ wave offset are subsequently detected with an improved version of Woody's method [8], so as to properly segment the TQ intervals. Their mean-correction and concatenation leads to the definition of the $(L \times N)$ matrix:

$$
\mathbf{Y}_{\mathrm{AA}}=\left[\mathbf{y}_{\mathrm{AA}}(1) \cdots \mathbf{y}_{\mathrm{AA}}(N)\right] \in \mathbb{R}^{L \times N}
$$

namely, the signal depicting AA content. Vector $\mathbf{y}_{\mathrm{AA}}(t)=$ $\left[y_{1}(t), \ldots, y_{L}(t)\right]^{T}$ represents the multilead AA signal measured at time sample $t$, and $N$ the number of samples of the AA signal $y_{\ell}(t)$ for each lead $\ell=1,2, \ldots, L$, with $L=12$ in the standard ECG.

\section{Multilead descriptor of $f$-wave amplitude}

Previous works have established that higher values of $f$ wave peak-to-peak amplitude on the surface ECG predict AF termination by CA [4]. Nonetheless, conventional approaches are affected by higher error probability, due to the manual computation of this variable. Moreover, they merely deal with a single electrode, so neglecting contributions provided from the whole ensemble of ECG leads. 
The ECG-based feature presented in [5] is able to condense the information about $\mathrm{f}$-wave spatial distribution over the ECG leads. First, the predictor proposed in [4] is computed in an automatic manner. An algorithm based on cubic spline interpolation of AA signal local extrema outputs the parameter:

$$
D\left(y_{\ell}\right)=\frac{1}{N} \sum_{t=1}^{N}\left(e_{\mathrm{MAX}}(t)-e_{\mathrm{MIN}}(t)\right)
$$

where $e_{\mathrm{MAX}}(t)$ and $e_{\mathrm{MIN}}(t)$ represent the upper and the lower envelopes passing through the local maxima and minima, respectively, of $y_{\ell}(t)$. Then, ECG inter-lead variability is accounted for thanks to a rank-1 approximation of the AA matrix $\mathbf{Y}_{\mathrm{AA}}$ determined by means of PCA. This technique decomposes a multivariate set of observations into a linear combination of maximum-variance sources, or principal components $(\mathrm{PCs}) x_{k}(t), k=1, \ldots, L$ :

$$
\mathbf{y}_{\mathrm{AA}}(t)=\mathbf{M x}(t)=\sum_{k=1}^{L} \mathbf{m}_{k} x_{k}(t) .
$$

They are stored in decreasing order of variance, so we expect that the first source can accurately approximate the input signal and characterize f-wave amplitude content over leads. By applying (2) on each row of the term $\mathbf{m}_{1} x_{1}$ and averaging all single-lead f-wave amplitude descriptors, we obtain the parameter $\bar{D}_{L}$ as the final output:

$$
\bar{D}_{L}=\frac{\sum_{\ell=1}^{L} d_{\ell}}{L}=\frac{\sum_{\ell=1}^{L}\left|m_{\ell 1}\right| D\left(x_{1}\right)}{L}=\frac{\left\|\mathbf{m}_{1}\right\|_{1}}{L} D\left(x_{1}\right)
$$

where $\|\cdot\|_{1}$ denotes the L1-norm operator. This index summarizes inter-lead f-wave amplitude distributions.

In [5], the role of $\bar{D}_{L}$ as a CA outcome predictor has been put in evidence: the higher its value, the more likely procedural AF termination. This result can be considered as a multilead extension of [4]. In the present work, a further role is ascribed to $\bar{D}_{L}$ as a descriptor of $\mathrm{AF}$ evolution during $\mathrm{CA}$, reflected on $\mathrm{f}$-wave amplitude temporal variations which are negative for successful procedures, positive otherwise, regardless of the observation period during follow-up.

\section{Statistical Analysis and Results}

Values of all parameters have been expressed as mean \pm standard deviation for each category. In Table I, subscripts SUCC and FAIL refer to successful and failing CA procedures, respectively, according to the protocols above presented, whereas subscripts ST and LT are related to the length of the observation blanking period (short-term and long-term period, respectively); headings START and END are associated with the moment of the acquisition of the ECG recordings during the procedure. For each patients' group (SUCC, FAIL) and follow-up window (ST, LT), the relation between $\overline{D_{L}}$ values computed at the starting of the ablation (independent variable $X$ ) and those obtained at its conclusion (dependent variable $Y$ ) is scrutinized, and Pearson's correlation coefficient $R$ is calculated to indicate
TABLE I: Statistical analysis and results

\begin{tabular}{|c||c||c||c||c||c|}
\hline & START & END & $R$ & $S$ & $P_{S}$ \\
\hline$\left(\bar{D}_{L}\right)_{\text {SUCC,ST }}$ & $0.049 \pm 0.070$ & $0.046 \pm 0.060$ & 0.982 & 0.836 & \multirow{2}{*}{$1.52 \cdot 10^{-18}$} \\
$\left(\bar{D}_{L}\right)_{\text {FAIL,ST }}$ & $0.022 \pm 0.010$ & $0.033 \pm 0.027$ & 0.900 & 2.422 & \\
\hline$\left(\bar{D}_{L}\right)_{\text {SUCC,LT }}$ & $0.055 \pm 0.100$ & $0.051 \pm 0.085$ & 0.998 & 0.849 & \multirow{2}{*}{$8.52 \cdot 10^{-5}$} \\
$\left(\bar{D}_{L}\right)_{\text {FAIL }, \mathrm{LT}}$ & $0.039 \pm 0.020$ & $0.036 \pm 0.023$ & 0.894 & 1.008 & \\
\hline \hline$D\left(V_{1}\right)_{\text {SUCC,ST }}$ & $0.068 \pm 0.022$ & $0.067 \pm 0.022$ & 0.789 & 0.802 & \multirow{2}{*}{$1.68 \cdot 10^{-2}$} \\
$D\left(V_{1}\right)_{\text {FAIL }, \mathrm{ST}}$ & $0.054 \pm 0.018$ & $0.048 \pm 0.012$ & 0.665 & 0.446 & \\
\hline$D\left(V_{1}\right)_{\text {SUCC,LT }}$ & $0.076 \pm 0.024$ & $0.070 \pm 0.023$ & 0.883 & 0.834 & \multirow{2}{*}{$1.12 \cdot 10^{-4}$} \\
$D\left(V_{1}\right)_{\text {FAIL }, \mathrm{LT}}$ & $0.054 \pm 0.012$ & $0.062 \pm 0.020$ & 0.840 & 1.392 & \\
\hline \hline NMSE $\left._{3}\right)_{\text {SUCC,ST }}$ & $15.4 \pm 15.9$ & $14.0 \pm 14.1$ & 0.092 & 0.082 & \multirow{2}{*}{$2.40 \cdot 10^{-3}$} \\
$\left(\mathrm{NMSE}_{3}\right)_{\mathrm{FAIL}, \mathrm{ST}}$ & $18.7 \pm 16.9$ & $35.5 \pm 29.7$ & 0.813 & 1.428 & \\
\hline $\left.\mathrm{NMSE}_{3}\right)_{\text {SUCC,LT }}$ & $9.80 \pm 9.65$ & $8.50 \pm 7.80$ & 0.476 & 0.384 & \multirow{2}{*}{$8.49 \cdot 10^{-1}$} \\
$\left(\mathrm{NMSE}_{3}\right)_{\mathrm{FAIL}, \mathrm{LT}}$ & $25.1 \pm 24.7$ & $26.5 \pm 28.9$ & 0.251 & 0.294 & \\
\hline
\end{tabular}

the degree of linear dependence between variables. The value of the slope $S$ of each regression law $Y=S X+I$ is examined so as to quantify CA effects over patients' heart substrate and their temporal dynamics, knowing that the intercept $I$ has small values. In addition, statistically significant differences between slope values have been assessed through a parallelism test for each couple of regression lines (SUCC, FAIL); $p$ values output by such test are referred to as $P_{S}$ in Table I.

The scatter plots in Figure 2 display the distribution of $\overline{D_{L}}$ values acquired after the completion of the ablation $\left({\overline{\left(D_{L}\right)}}_{\mathrm{END}}\right)$ as a function of those describing the beginning of the procedure $\left({\overline{\left(D_{L}\right)}}_{\mathrm{START}}\right)$ for each criterion of procedural success (short-term success and long-term success, respectively); the regression laws related to each category. Statistical analysis has been carried out under a confidence level $\alpha$ equal to 0.05 .

A comparison with previous works has been drawn as well. f-wave peak-to-peak amplitude $D\left(V_{1}\right)$ has been studied on lead $\mathrm{V}_{1}$ [4], [5]. Furthermore, the $\mathrm{NMSE}_{3}$ index introduced in [3] has been computed for each CA step on the same electrode and analyzed according to the protocol aforementioned as well.

\section{Discussion}

As shown in Tab. I, a highly linear correlation between $\bar{D}_{L}$ values concerning the beginning of the procedure and those acquired at its completion is exhibited. This result can be generalized for each class of subjects and CA outcome observation time. We assumed that successful CA procedures should result in a progressive decrease of f-wave amplitude values. Indeed, a significant reduction in f-wave amplitude is observed in patients experiencing procedural AF termination, underlined by a value of the slope $S$ less than unity. On the contrary, in the case of failing procedures, there is no effect on f-waves, whose amplitude raises or remains unchanged, as the disease holds up. Moreover, statistically significant differences are obtained for $S$ values related to each category of patients (SUCC, FAIL) at each time of follow-up. This shows the ability of our descriptor to quantify heart substrate changes performed by CA, which are specific for each group of subjects. The index rendering f-wave amplitude on $\mathrm{V}_{1}\left(D\left(V_{1}\right)\right)$ provides statistically significant inter-class differences, but its short-term value decreases after CA whether the procedure is effective or not. This outcome is not consistent with our initial assumption, as we expect a reduction in f-wave amplitude only in case of procedu- 
ral AF termination. Moreover, the linearity assumption is not as reliable as for our descriptor. Conversely, singlelead amplitude seems to distinguish between the categories considered (SUCC, FAIL) more accurately in the longterm follow-up than in the short-term one. However, its discrimination ability is clearly outperformed by that of our multilead descriptor, since differences between successful and failing procedures are quantified by lower $P_{S}$ values; linear correlation between the starting and the end of the ablation proves to be weaker for $D\left(V_{1}\right)$ as well. Regarding $\mathrm{NMSE}_{3}$, even though significant differences are reported for the short-term CA outcome examination, the linearity hypothesis proves to be quite weak, especially when dealing with successful procedures, as low $R$ values are output by regression analysis. Similar conclusions can be drawn by the analysis over longer periods, which reveals no significant differences between regression line slopes as well. Finally, the study is led only in $V_{1}$, the lead classically employed by cardiologists to study AF in surface ECG. Even if it is well known that this lead exhibits the largest ratio of atrial to ventricular signal amplitude [9], contributions from other leads are not analyzed at all by this classical approach. Some pertinent information is neglected, thus leading to an incomplete comprehension of the disease.

\section{Conclusions}

Our study has corroborated the ability of our parameter to quantitatively evaluate $\mathrm{CA}$ impact on $\mathrm{AF}$ dynamics during its performance through a multilead characterization of $\mathrm{f}$ wave amplitude variations. A linear correlation between the beginning and the end of the procedure has been established. In particular, a reduction in $\bar{D}_{L}$ values can be associated with successful procedures, thus showing CA efficacy in progressively suppressing f-waves and restore SR, regardless of the length of the postoperative blanking period. Our research is hindered by the lack of comparison with endocardial recordings and the limited size of our long-term database, whose evaluation is also affected by several confusing factors, e.g., effects of cardioversion and/or drugs, which can alter the final procedural outcome. Despite these limitations, we can conclude that AF pathophysiology is deeply influenced by CA performance, and its modifications can be quantitatively evaluated by analyzing f-wave amplitude variations in the surface ECG.

\section{REFERENCES}

[1] M. Haïssaguerre, P. Jaïs, D.C. Shah, A. Takahashi, M. Hocini, G. Quiniou, S. Garrigue, A. Le Mouroux, P. Le Metayer, and J. Clémenty. Spontaneous initiation of atrial fibrillation by ectopic beats originating in the pulmonary veins. N. Engl. J. Med., 339:659-665, 1998.

[2] M. Haïssaguerre, P. Sanders, M. Hocini, and et al. Catheter ablation of long-lasting persistent atrial fibrillation: critical structures for termination. Journ. of Card. Electr., 16(11), 2005.

[3] P. Bonizzi, O. Meste, V. Zarzoso, D. G. Latcu, I. Popescu, P. Ricard, and N. Saoudi. Atrial fibrillation disorganization is reduced by catheter ablation: A standard ECG study. In Proc. IEEE EMBS, pages 52865289, Buenos Aires, Argentina, Aug. 31 - Sept. 42010.

[4] I. Nault, N. Lellouche, S. Matsuo, and et al. Clinical value of fibrillatory wave amplitude on surface ECG in patients with persistent atrial fibrillation. J. Interv. Card. Electrophysiol., 26(1):11-19, Oct. 2009.
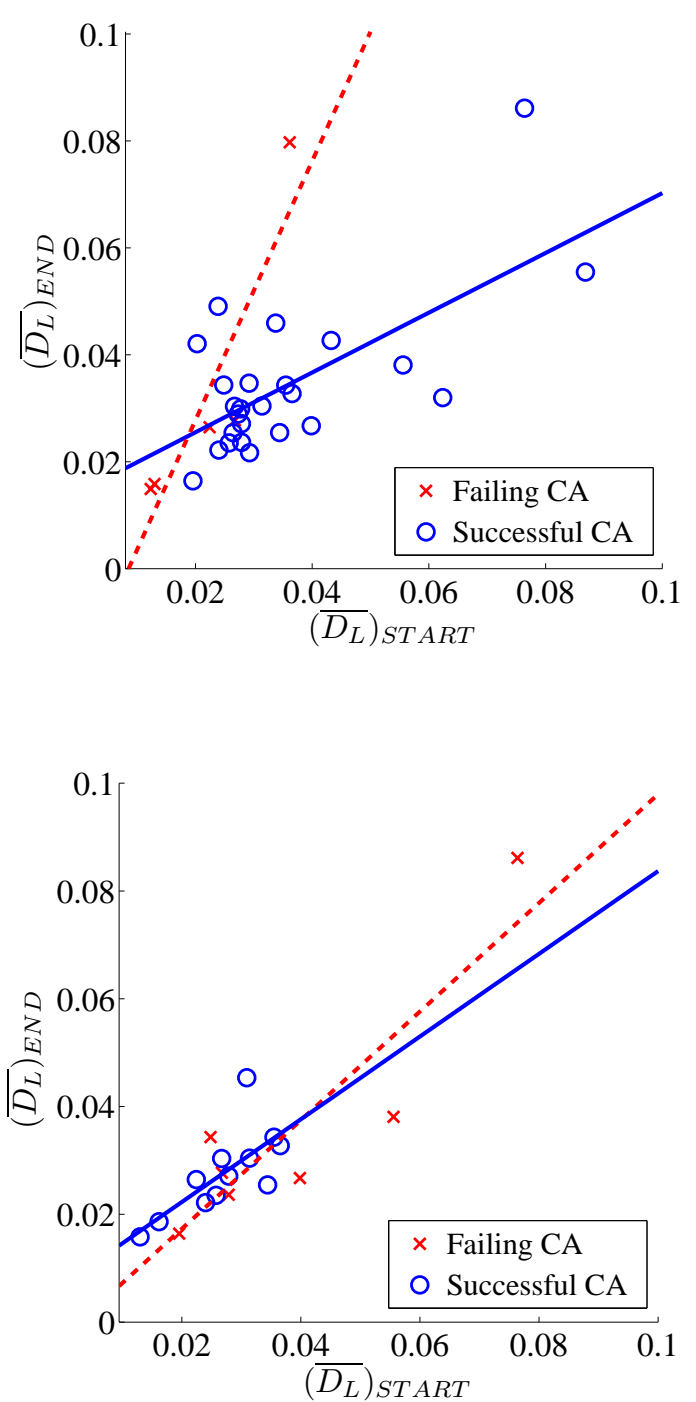

Fig. 2: Scatter plot of ${\overline{\left(D_{L}\right)}}_{\text {END }}$ as a function of ${\overline{\left(D_{L}\right)}}_{\text {START }}$ and data regression lines associated with each category (successful CA: O, continuous line; failing CA: $\times$, dotted line.). (Top) Short-term success; (Bottom) Long-term success.

[5] M. Meo, V. Zarzoso, O. Meste, D. G. Latcu, and N. Saoudi. Noninvasive prediction of catheter ablation outcome in persistent atrial fibrillation by exploiting the spatial diversity of surface ECG. In Proc. Annual Int. Engineering in Medicine and Biology Society, pages 55315534, Boston, USA, 2011.

[6] H. Calkins, J. Brugada, D. L. Packer, and et al. HRS/EHRA/ECAS expert consensus statement on catheter and surgical ablation of atrial fibrillation: recommendations for personnel, policy, procedures and follow-up. Europace, 9:335-379, 2007.

[7] J. Pan and W. J. Tompkins. A real-time QRS detection algorithm. IEEE Trans. Biomed. Eng., 3(3):230-236, 1985.

[8] A. Cabasson and O. Meste. Time delay estimation: A new insight into the woody's method. IEEE Signal Processing Letters, 15:573-576, 2008.

[9] S. Petrutiu, N.g. Jason, G.M. Nijm, and et al. Atrial fibrillation and waveform characterization. IEEE Eng. in Med. and Biol. Mag., 25(6):24-30, 2006. 